Received: 2016.05.24

Accepted: 2016.05.24

Published: 2016.05 .24

\section{Retraction note: Effects of Bariatric Surgery on Incidence of Obesity-Related Cancers}

\author{
Xiang-wu Yang \\ Peng-zhou Li \\ Li-yong Zhu \\ Shai-hong Zhu
}

\title{
Retraction Notice:
}

In the article entitled, "Effects of Bariatric Surgery on Incidence of Obesity-Related Cancers: A Meta-Analysis" which was published in Medical Science Monitor 2015;21: 1350-1357, sections in the text have been directly copied from a previously published article, entitled, "The Effects of Bariatric Surgery on Colorectal Cancer Risk: Systematic Review and Meta-Analysis", Sorena Afshar, Seamus B. Kelly, Keith Seymour, Jose Lara, Sean Woodcock, John C. Mathers in Obesity Surgery 2014; 24(10): 1793-1799. Thus owing to duplicity of text, the article is being retracted.

\section{Reference:}

1. Xiang-wu Yang, Peng-zhou Li, Li-yong Zhu, Shai-hong Zhu: Effects of Bariatric Surgery on Incidence of Obesity-Related Cancers: A Meta-Analysis. Med Sci Monit, 2015; 21: 1350-57; DOI 10.12659/MSM.893553 